\section{Establishment of Molecular Identity Cards for Cucumis melo Cultivars Using SSR Markers}

\author{
Li Li, Xiulan Xu, and Ping Wu \\ Beijing Vegetable Research Center, Beijing Academy of Agriculture and \\ Forestry Sciences, Key Laboratory of Biology and Genetic Improvement of \\ Horticultural Crops (North China), Ministry of Agriculture, Beijing 100097, \\ People's Republic of China; Key Laboratory of Urban Agriculture (North), \\ Ministry of Agriculture, Beijing 100097, People's Republic of China; and \\ Supervision, Inspection and Test Center of Vegetable Seed Quality, Beijing \\ Government, Beijing 100097, People's Republic of China
}

\section{Guo Zhang}

Tai'an Zhengtai Science and Technology Extension Center, Tai'an 271000, Shandong, China

\author{
Xiaobing Zhang ${ }^{1}$ \\ Shandong Institute of Pomology, Tai'an 271000, Shangdong, China
}

Additional index words. melon, Cucumis melo, SSR markers, DNA barcodes, molecular identity card, cultivar identification

\begin{abstract}
Twenty-four representative melon varieties and six parental cultivars were examined in this study. Among 159 pairs of simple sequence repeat (SSR) primers, 18 SSR core primers with rich polymorphic information, a large number of genotypes, and the ability to distinguish different melon varieties were selected. A total of 113 genotypes were detected among the 30 experimental materials, with an average of 6.28 genotypes for each pair of primers. The polymorphic information content was on average 0.6807 , ranging from 0.5618 to 0.7885 . Specific bands of the primers for the 30 experimental materials were analyzed, and by combining different primer loci, all 30 varieties were identified. Unique barcodes for molecular identity cards for the 30 experimental materials were established using the fingerprints formed with this SSR marker system. Each variety has a unique identity card that can be applied for the registration of the newly bred varieties, the protection of breeders' rights, and the authenticity of breeds after promulgation of the new Seed Law of the People's Republic of China.
\end{abstract}

Cucumis melo L., a cross-pollinated plant in the family Cucurbitaceae, is one of the top ten most popular fruits and is widely cultivated around the world. According to statistics of the Food and Agriculture Organization Corporate Statistical Database (FAOSTAT), melon production in China was 14.3368 million $t$ in 2013 , accounting for $48.66 \%$ of the world's production and making China the world's largest melon producer (Yang et al., 2016). Breeding of new melon varieties has also developed rapidly with the continuous expansion of the area for melon cultivation in China. In 2001-15, the number of new melon varieties identified by the National Committee

Received for publication 25 Sept. 2017. Accepted for publication 29 Nov. 2017.

This project is supported by the Beijing Academy of Agriculture and Forestry Found (JNKST201621), Beijing Academy of Agriculture and Forestry Finance Special Subject (KJCX20170102), and Beijing Academy of Agriculture and Forestry Found (KJCX20150202).

${ }^{1}$ Corresponding author. E-mail: zhangxiaobing766@ sina.com. for Appraisal of Crop Strains alone reached 53 (Industry Public Column, 2016), leading to an increasing number of cultivars in production per year and a large number of varieties approved in provincial committees. However, the repeated use of a set of excellent-backbone breeding cultivars has led to decreases in the genetic differences between some varieties because of this narrow genetic background. Therefore, it has become difficult to differentiate cultivars based on traditional morphological identification. In addition, melon seed dealers in the rural seed markets of China are mostly individual operators. Their management is relatively difficult to regulate, resulting in some individual seed dealers misrepresenting other melon seeds as the bestselling seeds and even arbitraging or selling piratical varieties bred by others. Furthermore, there is regular occurrence of the same variety of melon seeds being sold under different names as well as different varieties of melon seeds having the same name (Wang et al., 2010), which has caused significant loss of intellectual property rights to breeders as well as loss of production and cultivation to farmers.
Molecular marker technologies offer alternative approaches for achieving genetic diversity and identifying cultivars. For many molecular markers that have undergone rapid development, single-nucleotide polymorphisms (SNPs) are a good choice for marker-based studies of genetic diversity and cultivar identification (Deleu et al., 2009; Pavan et al., 2017). However, it is difficult to apply SNPs in most melon breeding programs because of the high cost and difficulties associated with SNP genotyping (Ding and Jin, 2009). This is particularly true for monitoring laboratories supported by local governments and small breeding laboratories in developing countries. Simple sequence repeat fingerprints offer obvious advantages, including rich polymorphism information, codominant inheritance, simple and easily performed experimental procedures, and clear experimental results that are easy to analyze, allowing for efficient and accurate detection of genetic variation in vegetable hybrids. With the development of expressed sequence tag-SSR and genomic SSR loci and markers in melons (Chiba et al., 2003; Fernandez-Silva et al., 2008; Fukino et al., 2007; Park et al., 2013; Seung and Yong, 2006), SSR markers have been increasingly applied for genetic diversity analysis of breeding parental cultivars and germplasm resources (Ajaz et al., 2014; Dhillon et al., 2009; Hu et al., 2015; Raghami et al., 2014; Roy et al., 2012; Zhang et al., 2017; Zhu et al., 2016; Weng, 2010), genetic map construction (Harel-Beja et al., 2010), and purity detection for melon hybrids (Cheng-Xiang et al., 2005). Moreover, with the discovery of the network and development of molecular biotechnology, Paul Hebert, a taxonomist at the University of Guelph in Canada, proposed the concept of DNA barcoding (Hebert et al., 2003a, 2003b), i.e., achieving rapid identification of an animal or plant species using a standard DNA sequence (Waugh, 2007; Yuan et al., 2009). As a newly developed technology, DNA barcoding has been gradually applied to species identification and genetic diversity analysis (Bucklin et al., 2010; Ekrem et al., 2007; Linares et al., 2009; Vogler and Monaghan, 2007), yet no application of DNA barcoding technology to the identification of vegetable varieties has been reported., Twenty-four representative varieties on the market and three self-bred thick-skinned melon hybrid varieties and their parents, provided by Tai'an Zhengtai Technology Extension Center, were used in this study as research materials, and their fingerprints were established using the SSR technique to construct unique barcodes (or two-dimensional codes) as identity markers, i.e., molecular identity cards. This system will provide data at the molecular level for authenticity in melon variety identification. In addition, the data can be used for the protection of intellectual property rights, for rapid molecular identification of melon hybrids and their parents, and for the declaration of new varieties of melon as well as the registration 
of newly bred varieties after the promulgation and implementation of the new seed law. Furthermore, this technique will provide a theoretical basis for the preservation and selection of melon breeding cultivars.

\section{Materials and Methods}

Materials. Seeds of the experimental melon varieties [C. melo ssp. Conomon (Thunb.) Greb] used as research materials were collected from 24 representative varieties on the market and three self-bred thickskinned melon hybrid varieties and their parents were provided by Tai'an Zhengtai Technology Co., Ltd. (Tai'an, Shandong).
Information on these sample cultivars is shown in Table 1. Experiments were carried out in 2016 at the Supervision, Inspection and Test Center of Vegetable Seed Quality of Beijing and the Molecular Detection Laboratory of Seed Technology of the Vegetable Research Center, Beijing Academy of Agriculture and Forestry Sciences.

Preparation of DNA. Young leaves on mature plants, young true leaves on strongly growing shoots (50-60 mg), or seedlings within $5 \mathrm{~d}$ after germination were collected and placed in a $1.5-\mathrm{mL}$ centrifuge tube for DNA extraction. The DNA extraction method was modified using sodium dodecyl sulfate (Li and Zheng, 2009).

Table 1. Information of Cucumis melo materials and molecular IDs.

\begin{tabular}{|c|c|c|c|c|c|}
\hline Number & Name $^{z}$ & Type & Source & Character & Molecular ID \\
\hline 1 & TaiWanMeiYu & $\mathrm{H}$ & Henan & Thick-skinned & 020501050202 \\
\hline 2 & JingYu No. 1 & $\mathrm{H}$ & Beijing & Thick-skinned & 030303030202 \\
\hline 3 & TianGuan No. 9 & $\mathrm{H}$ & Beijing & Thick-skinned & 020323450102 \\
\hline 4 & Youshawang & $\mathrm{H}$ & Beijing & Thick-skinned & 030303050202 \\
\hline 5 & Elissbeth No. 3 & $\mathrm{H}$ & Zhejiang & Thick-skinned & 030323450102 \\
\hline 6 & TunFengLvTian & $\mathrm{H}$ & Anhui & Thin-skinned & 020301010304 \\
\hline 7 & FengTian No. 11 & $\mathrm{H}$ & Hefei & Thick-skinned & 020201010203 \\
\hline 8 & JapanTianBao & $\mathrm{H}$ & Henan & Thin-skinned & 030302020303 \\
\hline 9 & F377 & $\mathrm{H}$ & Henan & Thick-skinned & 020201010202 \\
\hline 10 & ZhongTianXueHong & $\mathrm{H}$ & Henan & Thick-skinned & 020301340102 \\
\hline 11 & ZhongTianZhongTian No. 2 & $\mathrm{H}$ & Henan & Thick-skinned & 030301010202 \\
\hline 12 & ZhongTianWangLuoShiDai & $\mathrm{H}$ & Henan & Thick-skinned & 030303030202 \\
\hline 13 & ZhongTianN1 & $\mathrm{H}$ & Henan & Thick-skinned & 030423050204 \\
\hline 14 & F347 & $\mathrm{H}$ & Henan & Thick-skinned & 040402020202 \\
\hline 15 & ZhengTaiWangWenN5 & $\mathrm{H}$ & Shandong & Thick-skinned & 030301010103 \\
\hline 16 & ZhengTaiWangWenN5M & $\mathrm{L}$ & Shandong & Thick-skinned & 030301010101 \\
\hline 17 & ZhengTaiWangWenN5F & $\mathrm{L}$ & Shandong & Thick-skinned & 030301010202 \\
\hline 18 & GuiFei & $\mathrm{H}$ & Shandong & Thick-skinned & 030402040103 \\
\hline 19 & GuiFeiM & $\mathrm{L}$ & Shandong & Thick-skinned & 040401010101 \\
\hline 20 & GuiFeiF & $\mathrm{L}$ & Shandong & Thick-skinned & 030301010202 \\
\hline 21 & WuYueDuZun & $\mathrm{H}$ & Shandong & Thick-skinned & 040401010103 \\
\hline 22 & WuYueDuZunM & $\mathrm{L}$ & Shandong & Thick-skinned & 030301010202 \\
\hline 23 & WuYueDuZunF & $\mathrm{L}$ & Shandong & Thick-skinned & 030302020101 \\
\hline 24 & JingYuYangJiaoSu & $\mathrm{H}$ & Beijing & Thin-skinned & 010101340404 \\
\hline 25 & JingYuLvBao & $\mathrm{H}$ & Beijing & Thin-skinned & 030402450304 \\
\hline 26 & JingYu30 & $\mathrm{H}$ & Beijing & Thin-skinned & 010301450404 \\
\hline 27 & JingYu268 & $\mathrm{H}$ & Beijing & Thick-skinned & 010301450203 \\
\hline 28 & JingYu357 & $\mathrm{H}$ & Beijing & Thin-skinned & 040401450404 \\
\hline 29 & LvMaGua & $\mathrm{H}$ & Henan & Thin-skinned & 010103030303 \\
\hline 30 & HuangCuiMi & $\mathrm{H}$ & Henan & Thin-skinned & 034504050404 \\
\hline
\end{tabular}

${ }^{\mathrm{z}}$ The abbreviations of the samples in Fig. 1 are derived from the initial consonants of the sample name. $\mathrm{L}=$ inbred line; $\mathrm{H}=$ hybrid. Molecular IDs represent the size of the gene fragment listed in Table 4.

Primer screening for SSR markers. A total of 159 pairs of SSR primer sequences from Cucumis were selected (Cheng-Xiang et al., 2005; Chiba et al., 2003; Harel-Beja et al., 2010; Seung and Yong, 2006; Song et al., 2012) for synthesis at SANGON Biotech Co., Ltd. (Shanghai, China) and SBS Genetech Co., Ltd. (Beijing, China). Twenty-four representative melon hybrids on the melon seed market and six parental cultivars (Table 1) were selected for amplification using these primers. Eighteen pairs of primers showing clear bands with good reproducibility, rich polymorphic information, and the ability to detect at least four alleles were selected as the core primers (Table 2).

Polymerase chain reaction (PCR) amplification and detection by electrophoresis. PCR reaction system: The reaction included $0.4 \mu \mathrm{L}$ of $10 \mathrm{mmol} \cdot \mathrm{L}^{-1}$ deoxynucleotides, $2 \mu \mathrm{L}$ of $10 \times$ PCR buffer, $0.15 \mu \mathrm{L}$ of $5 \mathrm{U} \cdot \mu \mathrm{L}^{-1} 10 \mathrm{Taq}$ enzyme, $2 \mu \mathrm{L}$ of $60-200 \mathrm{ng} \cdot \mu \mathrm{L}^{-1}$ template DNA, $2 \mu \mathrm{L}$ of forward primer, $2 \mu \mathrm{L}$ of reverse primer, and water to a total volume of $20 \mu \mathrm{L}$. The PCR program was as follows: $94{ }^{\circ} \mathrm{C}$ for $5 \mathrm{~min}$; 35 cycles of $94{ }^{\circ} \mathrm{C}$ for $15 \mathrm{~s}, 55^{\circ} \mathrm{C}$ for $15 \mathrm{~s}$, and $72{ }^{\circ} \mathrm{C}$ for $30 \mathrm{~s}$; and $72{ }^{\circ} \mathrm{C}$ for $4 \mathrm{~min}$. The samples were stored at $4{ }^{\circ} \mathrm{C}$. The amplification reaction was performed using a PE9700 amplification apparatus from Gene Company Ltd. (Beijing, China). The amplification products were subjected to electrophoresis on an ultrahigh-resolution 3\% agarose gel from Amresco, Inc. (Solon Industrial Pkwy) containing $0.5 \mathrm{mg} \cdot \mathrm{mL}^{-1}$ GoodView from SBS Genetech Co., Ltd. Imaging was performed using a Syngene gel-imaging apparatus produced by Gene Company Ltd.

SSR fingerprint data. The 0, 1 system was used to record the presence and absence of different-sized bands for SSR loci: the presence of an amplification band for each pair of primers was recorded as 1; absence was recorded as 0 . The bands obtained from amplification of the 30 sample cultivars using the 18 pairs of primers were recorded using the 0,1 system in an Excel table to establish an SSR map. After converting the information into a text file, the data were input into ClusterProject software for analysis, forming the pattern of the 30 cultivars shown in Fig. 1.

Table 2. Basic information of the 18 core primer pairs.

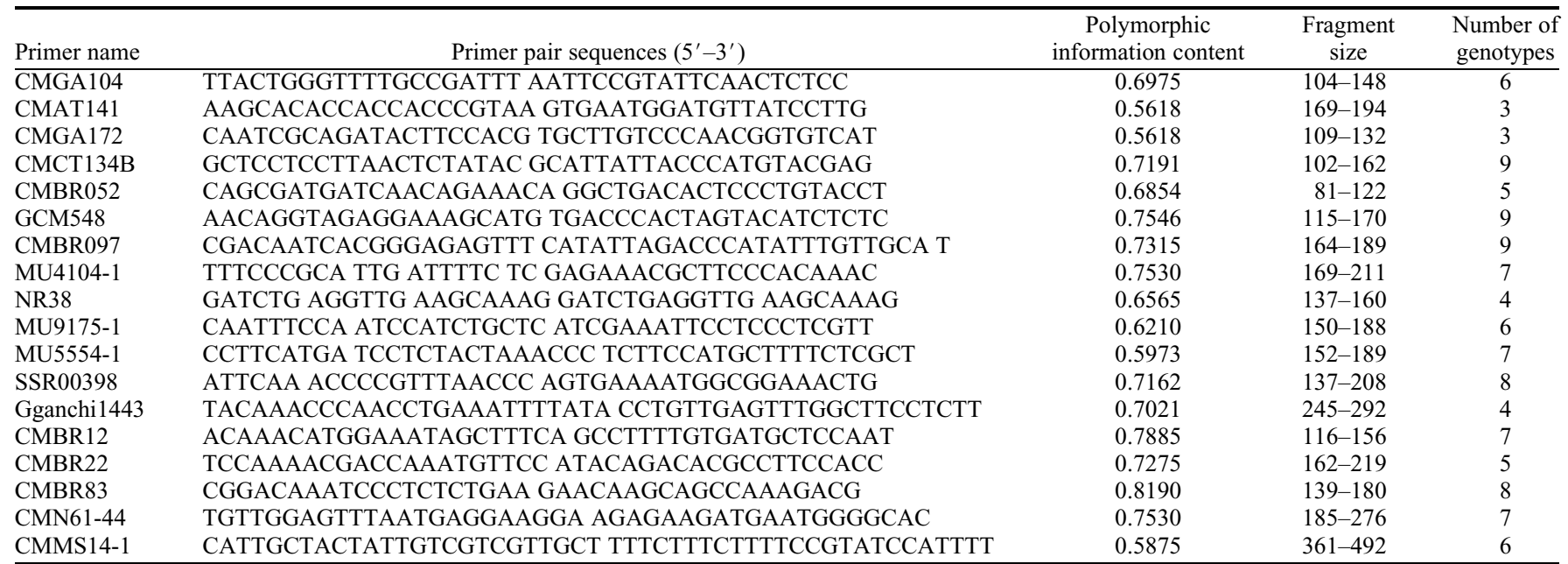




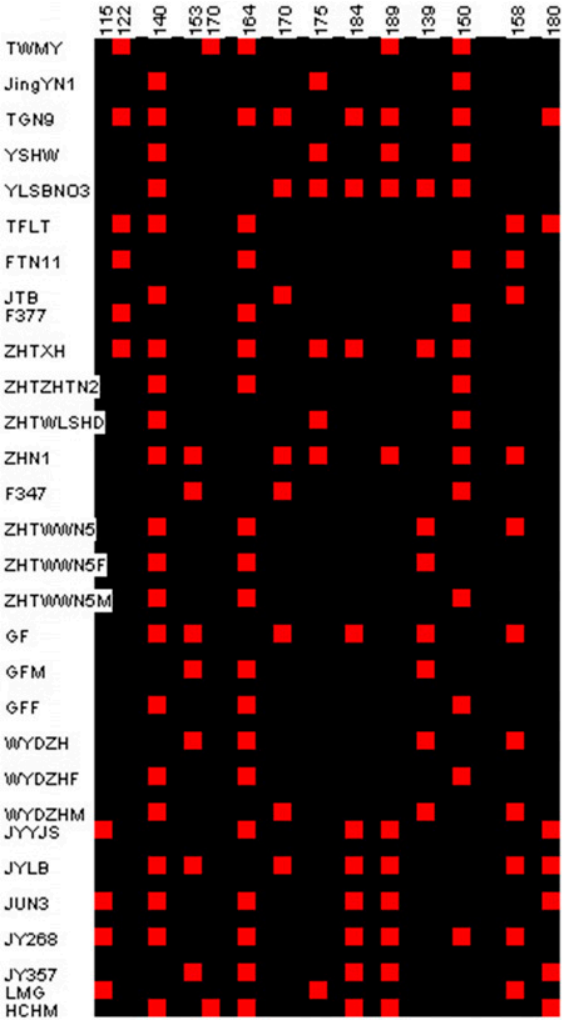

Fig. 1. Simple sequence repeat (SSR) fingerprint pattern for the 30 Cucumis melo cultivars. On the left of the figure are the names of the 30 samples. Abbreviations are shown in Table 1. The top of the figure shows the size of the fragments of different genes amplified by three primers. Fragments 164, 170, 175, 184, and 189 bp are amplified by primer CMBR097; fragments $115,122,140,153$, and $170 \mathrm{bp}$ are amplified by primer GCM548; and fragments $139,150,158$, and $180 \mathrm{bp}$ are amplified by primer CMBR83. Red denotes presence; black denoted absence.
The formula for calculating the polymorphic information content was

$$
\mathrm{PIC}=1-\sum_{i=1}^{n} P_{i}^{2}-\sum_{i=1}^{n-1} \sum_{j=i+1}^{n} 2 P_{i}^{2} P_{j}^{2},
$$

where $n$ is the total number of alleles detected for a locus of a marker, $P_{i}$ is the frequency of the ith allele in the set of genotypes investigated, and $P_{j}$ is another frequency of the $j$ th allele in the set of genotypes investigated.

\section{Results}

Polymorphism in SSR primer amplification. After amplification of the 30 melon samples using the 159 pairs of primers, 18 pairs of primers showing clear bands, good reproducibility, and high polymorphic information content were selected as core primers for the melons (Table 2). DNA for the 24 melon hybrids and six parental cultivars was amplified using these 18 pairs of primers, resulting in 113 detectable genotypes, with an average number of genotypes for each pair of primers of 6.28 (ranging from 3 to 9). The differences among the SSR product fragments obtained from the amplification of different melon germplasms reflect the differences among the melon varieties. The number of genotypes and the polymorphic information content for the 18 pairs of primers based on the amplification of the 30 melon varieties are shown in Table 2. The highest number of genotypes amplified by primer GCM548 was nine, with fragments ranging in size from 115 to $170 \mathrm{bp}$ and the highest polymorphic information content of 0.7546; the fingerprints with gel electrophoresis are shown in Fig. 2A. The second highest number of genotype loci was eight, which were detected using primer CMBR83; the amplified fragments ranged from 139 to $180 \mathrm{bp}$, and the highest polymorphic information content was 0.8190 ; the fingerprints with gel electrophoresis are shown in Fig. 2B. Nine pairs of primers have more genotypes than the average, accounting for $50 \%$ of all selected primers, and 12 pairs have a higher polymorphic information content than the average, accounting for $66.7 \%$ of all selected primers. More detected genotypes and higher polymorphic information content can reflect more differences among the varieties. The number of primers detecting genotypes was higher than the average and the polymorphic information content of the following primers was also higher than the average: MU4104-1, CMCT134B, CMBR12, SSR00398, CMBR83, CMN61-44, CMBR097, and GCM548. Among the 18 pairs of primers, the number of primer pairs in a combination that could identify the 30 melon cultivars ranged between two and five, with primer CMBR097 detecting nine genotype loci and distinguishing five cultivars, resulting in the highest identification rate. However, it was difficult to identify all of the cultivars by using only one pair of primers. Nonetheless, the primers with a high polymorphic information content and a large number of detected genotypes were of great value for constructing molecular identity cards for melon cultivars.

Screening of primer combinations and construction of SSR fingerprints. The general set of primers was divided into 30 varieties for testing in several groups; however, it was difficult for the primers to identify each variety. Each variety can exhibit a unique SSR fingerprint, forming a unique molecular identity card for authenticity identification. Regardless, the selection of primer loci should meet the requirement that the minimum number of primers is used to distinguish most melon varieties, thereby avoiding the
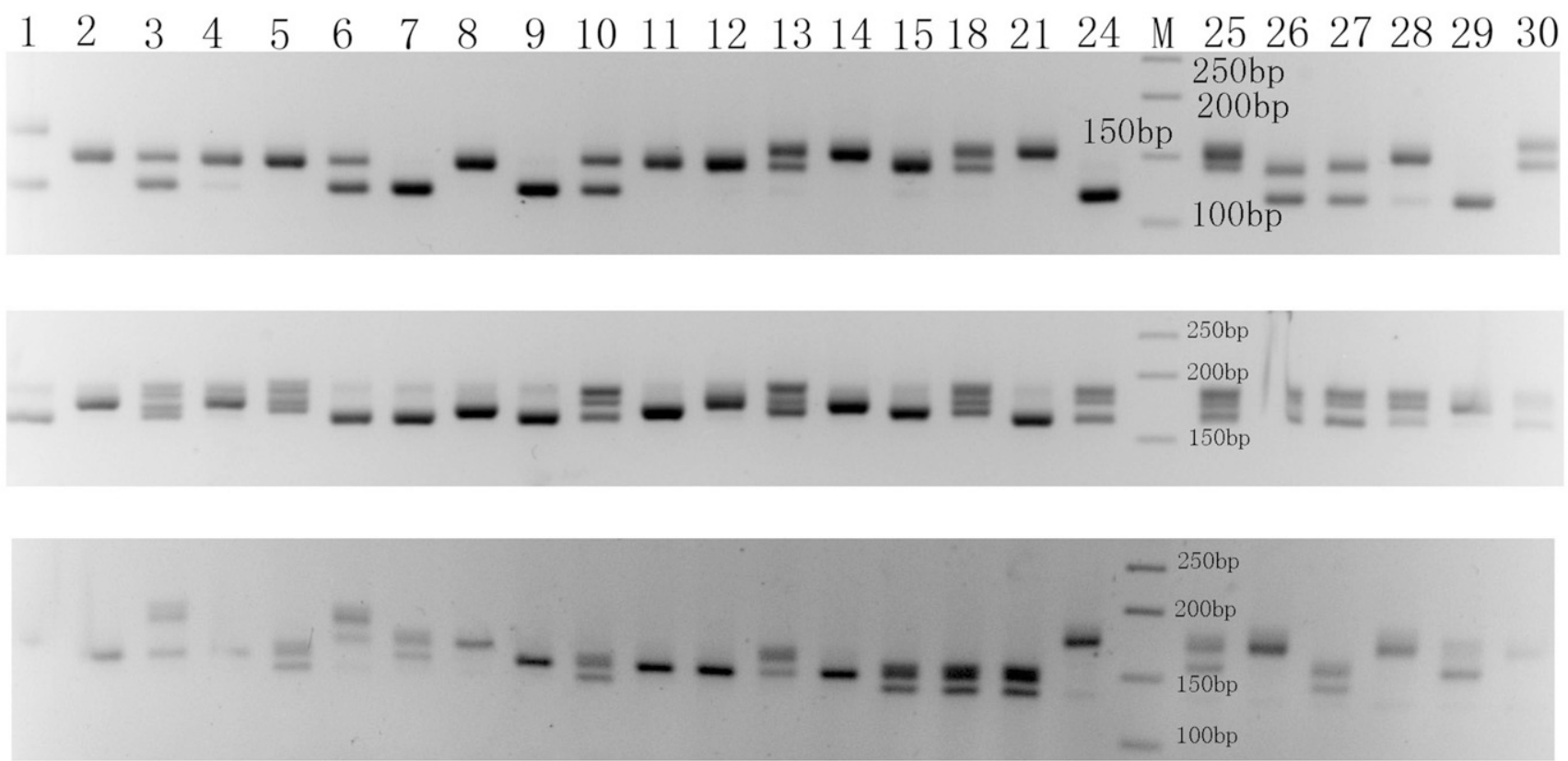

Fig. 2. Simple sequence repeat (SSR) profile for 24 varieties amplified by primers GCM548 (A), CMBR097 (B), and CMBR83 (C). The number represents the sample name listed in Table 1. M is Standard molecular weight with size labeled. 
Table 3. The 30 Cucumis melo varieties separately distinguished by combinations of primer pairs.

\begin{tabular}{cllc}
\hline Number & Primer combination & \multicolumn{1}{c}{ Cultivars can be distinguished ${ }^{z}$} & Identification rate (\%) \\
\hline 1 & CMBR12, MU4104-1 & $1,9,11,13,18,20,26,30$ & 26.67 \\
2 & CMBR12, CMN61-44 & $3,4,5,7,8,9,10,11,12,13,15,18,20,21,26,27$ & 53.33 \\
3 & CMBR12, CMBR83 & $3,4,5,8,9,10,11,13,18,26,29$ & 36.67 \\
4 & CMBR12, GCM548 & $1,4,8,11,13,14,15,18,19,21,26,27,28,30$ & 46.67 \\
5 & CMBR12, CMBR097 & $1,3,4,5,8,10,11,13,14,15,21,23,25,26,29$ & 50.00 \\
6 & MU4104-1, CMN61-44 & $3,5,7,9,10,11,15,16,18,21,26,30$ & 30.00 \\
7 & MU4104-1, CMBR83 & $3,6,7,8,11,26,27,29,30$ & 33.33 \\
8 & MU4104-1, GCM548R & $1,6,14,18,19,20,21,26,28,30$ & 36.67 \\
9 & MU4104-1, CMBR097 & $1,3,4,5,10,13,23,25,26,29,30$ & 40.00 \\
10 & CMN61-44, CMBR83 & $3,5,7,9,10,13,15,16,18,20,21,27$ & 60.00 \\
11 & CMN61-44, GCM548 & $1,3,6,7,9,10,13,14,15,18,19,20,21,25,26,27,28,30$ & 53.33 \\
12 & CMN61-44, CMBR097 & $1,2,3,4,5,8,9,10,13,14,18,20,21,23,25,27$ & 63.33 \\
13 & CMBR83, GCM548 & $1,3,5,6,7,8,9,10,13,14,18,19,24,25,26,27,28,29,30$ & 46.67 \\
14 & CMBR83, CMBR097 & $1,3,4,5,6,7,8,10,13,14,23,25,27,29$ & 53.33 \\
15 & GCM548, CMBR097 & $1,3,4,5,6,10,13,14,18,19,21,24,25,28,29,30$ & \\
\hline
\end{tabular}

${ }^{\mathrm{z}}$ The number represents the sample name listed in Table 1. The primer combination of CMBR83 and GCM548 distinguished the most varieties among 30 cultivars.

formation of unnecessarily complicated molecular identity cards. Accordingly, the primer combination was established based on a stepwise increase in the number of primers for screening the primer combinations that were able to distinguish all of the melon samples.

The primers resulting in a higher than average number of detected genotypes and polymorphic information content included MU4104-1, CMCT134B, CMBR12, SSR00398, CMBR83, CMN61-44, CMBR097, and GCM548. The six primers (MU4104-1, CMBR12, CMBR83, CMN61-44, CMBR097, and GCM548) with a high polymorphism information content and high number of detected genotypes were grouped in combinations of two to test their identification rate for the 30 melon varieties. The primer combination of CMBR83 and GCM548 showed the highest identification rate of $63.33 \%$, with the ability to identify 19 varieties. The CMN61-44 and GCM548 primer combination had the second highest identification rate, at $60.00 \%$, with the ability to identify 18 varieties. The next primer combinations were CMBR12 and CMN61-44, CMN61-44 and CMBR097, and GCM548R and CMBR097, with identification rates of $53.33 \%$ and the ability to identify 16 varieties (Table 3).

According to the identification results of pairwise combinations of primers, two primer pairs (CMBR83 and GCM548 and CMN61-44 and GCM548) with a high identification rate were selected. When three primers were grouped in combination, all 30 melon varieties were identified. The combination of three primers with the three highest polymorphic information contents and the three highest numbers of genotypes was GCM548, CMBR097, and CMBR83; the fingerprints with gel electrophoresis are shown in Fig. 2A-C. The primer combination of CMN61-44, GCM548, and CMBR097 exhibited the four highest polymorphic information contents and the four highest numbers of genotypes. Both of these combinations of three primers were able to generate the fingerprints of the 30 melon varieties; the fingerprint patterns of the 30 tested samples at the CMBR83, GCM548, and CMBR097 loci are shown in Fig. 1.
Coding of the molecular identity card for the varieties. The alleles of the combination of CMBR83, GCM548, and CMBR097 primers, which were able to distinguish all 30 melon varieties, were arranged in an ascending order and numbered from 01 (Table 4). For example, four alleles were detected after amplifying DNA from the 30 melon varieties using the primer CMBR83, in which the smallest fragment was $139 \mathrm{bp}$ and the largest $180 \mathrm{bp}$. In contrast, loci showing no genotype were recorded as -9 . The genotypes were arranged in ascending order and assigned a value from 01, e.g., the 139-bp genotype was assigned 01 and the 180-bp genotype was assigned 04 . The locus recorded as -9 was assigned 05 . The genotypes obtained at each of the three primer loci were encoded according to the assigned value to form a unique string for each sample (Table 1). For example, the genotypes of WuYueDuZun at the loci of CMBR83, GCM548, and CMBR097 were 139/158, 140, and $164 \mathrm{bp}$ and the corresponding assigned values were $01 / 03,04 / 04$, and $01 / 01$, forming the ordered string of 040401010103 . The string based on the order of loci by the same primers was converted into a unique barcode (i.e., a molecular identity card) for each variety using barcoding technology. For example, the barcode of the molecular identity card for WuYueDuZun is shown in Fig.3.

\section{Discussion}

To obtain a set of stable and reliable core primers, 24 representative melon cultivars available on the market and six parental varieties were collected in this study; 159 primers were screened to identify 18 pairs of core primers based on the number of detected genotypes and the richness of the detected polymorphism information content. This approach achieves the goal of distinguishing the most varieties using the smallest number of primers, from which molecular identity card information of the melon varieties can be constructed. A single pair of primers can divide the 30 varieties of melon into only several groups and cannot differentiate each individual variety. A combination of two pairs
Table 4. Rules for assigning values to alleles.

\begin{tabular}{lccc}
\hline & \multicolumn{3}{c}{ Primer } \\
\cline { 2 - 4 } Code $^{z}$ & CMBR097 & GCM548 & CMBR83 \\
\hline 1 & 164 & 115 & 139 \\
2 & 170 & 122 & 150 \\
3 & 175 & 140 & 158 \\
4 & 184 & 153 & 180 \\
5 & 189 & 170 & -9 \\
6 & -9 & -9 & \\
\hline
\end{tabular}

${ }^{\mathrm{z}}$ The code number represents different fragment sizes amplified by primers CMBR097, GCM548, and CMBR83.

of primers can identify a number of varieties, whereby specific combinations of two pairs of primers with high numbers of detected genotypes and high polymorphic information contents will significantly improve identification rates. Moreover, primer combinations containing three pairs of primers can completely identify all 30 varieties. Thus, the selection of appropriate primers is an important condition for establishing SSR fingerprints of melon varieties and for further constructing molecular identity cards.

In this study, 18 pairs of selected core primers showed high polymorphic information contents and were able to detect the different genotypes of melon cultivars examined. A primer combination with three pairs of primers was used to distinguish all 30 samples. However, with the continuously increasing number of cultivars available, some samples may have the same SSR loci and would thus be difficult to distinguish, e.g., parent-offspring samples with little difference between them. In this study, 159 pairs of SSR molecular marker primers were tested to accumulate an abundance of basic data for different primers. The primers were screened using various types of hybrids and inbred melon samples to obtain differential fingerprints of various melon samples. This set of core primers was able to achieve the goal of distinguishing the different varieties of melon with a high specificity using the smallest number of primer pairs. This method is easy to operate and highly efficient. It should be noted that when a new variety is added to the sample set, it is not necessary to retest the samples already tested; the 


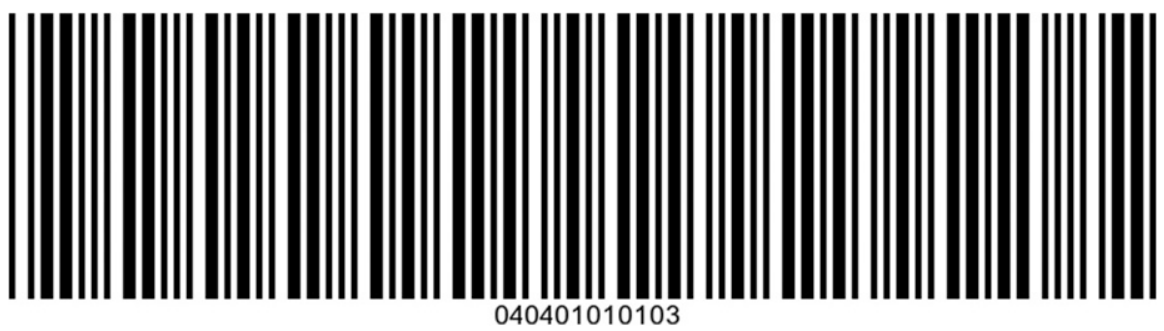

Fig. 3. Barcode of the molecular identity card for WuYueDuZhun.

established basic molecular data of the varieties can be effectively applied. That is, these 18 pairs of core primers can be directly used to amplify samples from new varieties, resulting in additional SSR fingerprint data.

DNA fingerprinting is the basis for the construction of molecular identity cards. However, a credible digital fingerprint (i.e., the molecular identity card) can be obtained only if a reliable electrophoretic fingerprint that can distinguish among different varieties is available. A molecular identity card is a unique identity that can be simply and intuitively assigned to each genetic variety. In this study, specific primers were screened based on SSR fingerprints; the alleles amplified by each pair of primers were arranged in ascending order and assigned Arabic numerals starting from 01 . A series of obtained bands are encoded by these numbers, forming a set of band coding data in the order of the primers as the molecular identity card of the variety. The allele obtained at the specific locus of each sample was encoded according to the assigned number to obtain a unique string. Next, barcoding technology was used to convert the unique string into a barcode logo, i.e., the barcode molecular identity card. This barcode molecular identity card can rapidly identify a variety using barcode scanning, thereby avoiding the trouble of manually reading the molecular identity card in string format. The obtained DNA fingerprint information can be converted into a unique molecular identity card for each sample using barcoding technology, thereby converting the molecular differences among different melon varieties into visible differences that can be quickly recognized by a machine and preventing the influence of environmental and human factors.

Applying this research, it may be possible to manage the supervision of illegal sales of fake seeds in the Chinese melon seed market to obtain greater profit. Some of the current issues include the following: 1) seed dealers sell muskmelon varieties with the same parentage under different trade names; 2) some varieties with different parentage carry the same name; and 3) some pure lines are labeled "hybrid" for commercial sale. The intellectual property rights of breeders would be protected, and the risk of great losses to farmers related to production and planting might be reduced. Thus far, the application of this research to crop seeds has not been reported.
Undoubtedly, the construction of this molecular identity card system for melon varieties has important research significance and application value in establishing a molecular database of melon germplasm resources. In practice, a number of samples with legal rights have been identified since 2013. The SSR profiles of Wuyueduzun, GuiFei, and ZhenTaiWangWen No. 5 have been registered. In the past 5 years, more than 500 melon samples have been tested for their hybrid purities in our laboratory. Additional benefits include the process of identifying and registering new melon varieties, protecting the rights and interests of breeders, ensuring the benefit to farmers of melon production and cultivation, and preventing fake and shoddy seeds from entering the seed market.

\section{Literature Cited}

Ajaz, A.M., V.K. Vashisht, K. Singh, A. Sharma, D.K. Singh, H. Singh, A.J. Monforte, J.D. McCreight, and N.P.S. Dhillon. 2014. Diversity among melon (Cucumis melo L.) landraces from the Indo-Gangetic plains of India and their genetic relationship with USA melon cultivars. Genet. Resources Crop Evol. 61:1189-1208.

Bucklin, A., R.R. Hopcroft, K.N. Kosobokova, L.M. Nigro, B.D. Ortman, R.M. Jennings, and C.J. Sweetman. 2010. DNA barcoding of Arctic Ocean holozooplankton for species identification and recognition. Deep Sea Res. Part II Top. Stud. Oceanogr. 57:40-48.

Cheng-Xiang, A., Y. Xian-Mei, L. Qing-Zhong, L. Zhi-Xin, M. Guo-Bin, and L. Lu. 2005. Application of SSR markers in testing the genetic purity of F1 hybrids of melon. J. Genet. Breed. 59:205-212.

Chiba, N., K. Suwabe, T. Nunome, and M. Hirai 2003. Development of microsatellite markers in melon (Cucumis melo L.) and their application to major cucurbit crops. Breed. Sci. 53:21-27.

Deleu, W., C. Esteras, C. Roig, M. Gonzalez-To, I. Fernandez-Silva, D. Gonzalez-Ibeas, J. Blanca, M.A. Aranda, P. Arus, F. Nuez, A.J. Monforte, M.B. Pico, and J. Garcia-Mas. 2009. A set of EST-SNPs for map saturation and cultivar identification in melon. BMC Plant Biol. 9:90.

Dhillon, N., J. Singh, M. Fergany, A.J. Monforte, and A. Sureja. 2009. Phenotypic and molecular diversity among landraces of snapmelon (Cucumis melo var. momordica) adapted to the hot and humid tropics of eastern India. Plant Genet. Resources Utilization 7:291300 .

Ding, C. and S. Jin. 2009. High-throughput methods for SNP genotyping. Methods Mol. Biol. 57:245-254.

Ekrem, T., E. Willassen, and E. Stur. 2007. A comprehensive DNA sequence library is essential for identification with DNA barcodes. Mol. Phylogenet. Evol. 43:530-542.

Fernandez-Silva, I., I. Eduardo, J. Blanca, C. Esteras, B. Pico, F. Nuez, P. Arus, J. Garcia-Mas, and A.J. Monforte. 2008. Bin mapping of genomic and EST-derived SSRs in melon (Cucumis melo L.). Theor. Appl. Genet. 118:139-150.

Fukino, N., Y. Sakata, M. Kunihisa, and S. Matsumoto. 2007. Characterisation of novel simple sequence repeat (SSR) markers for melon (Cucumis melo L.) and their use for genotype identification. J. Hort. Sci. Biotechnol. 82:330-334.

Harel-Beja, R., G. Tzuri, V. Portnoy, M. LotanPompan, S. Lev, S. Cohen, N. Dai, L. Yeselson, A. Meir, S.E. Libhaber, E. Avisar, T. Melame, P. van Koert, H. Verbakel, R. Hofstede, H Volpin, M. Oliver, A. Fougedoire, C. Stalh, J. Fauve, B. Copes, Z. Fei, J. Giovannoni, N. Ori, E. Lewinsohn, A. Sherman, J. Burger, Y. Tadmor, A.A. Schaffer, and N. Katzir. 2010. A genetic map of melon highly enriched with fruit quality QTLs and EST markers, including sugar and carotenoid metabolism genes. Theor. Appl. Genet. 121:511-533.

Hebert, P.D., A. Cywinska, and S.L. Ball. 2003a. Biological identifications through DNA barcodes. Proc. Biol. Sci. 270:313-321.

Hebert, P.D.N., S. Ratnasingham, and J.R. deWaard. 2003b. Barcoding animal life: Cytochrome $\mathrm{C}$ oxidase subunit 1 divergences among closely related species. Proc. Biol. Sci. 270:S96-S99.

$\mathrm{Hu}$, J., P. Wang, Y. Su, R. Wang, Q. Li, and K. Sunday. 2015. Microsatellite diversity, population structure, and core collection formation in melon germplasm. Plant Mol. Biol. Rpt. 33:439-447.

Industry Public Column. 2016. Introduction for some melon varieties that were identified by the national watermelon and melon varieties' trial. China Cucurbits Veg. 29(9):10-21 (Catalog list).

Li, L. and X. Zheng. 2009. The development of multiplex EST-SSR markers to identification Chinese cabbage [Brassica campestris L. chinensis (L.) makino and Brassica campestris L. Pekinensis (lour.) olsson] cultivars. Yuan Yi Xue Bao 36:1627-1634.

Linares, M.C., I.D. Soto-Calderon, D.C. Lees, and N.M. Anthony. 2009. High mitochondrial diversity in geographically widespread butterflies of Madagascar: A test of the DNA barcoding approach. Mol. Phylogenet. Evol. 50:485-495.

Park, I., J. Kim, J. Lee, S. Kim, O. Cho, K. Yang, J. Ahn, S. Nahm, and H. Kim. 2013. Development of SSR markers by next-generation sequencing of Korean landraces of chamoe (Cucumis melo var. makuwa). Mol. Biol. Rpt. 40:6855-6862.

Pavan, S., A.R. Marcotrigiano, E. Ciani, R. Mazzeo, V. Zonno, V. Ruggieri, C. Lotti, and L. Ricciardi. 2017. Genotyping-by-sequencing of a melon (Cucumis melo L.) germplasm collection from a secondary center of diversity highlights patterns of genetic variation and genomic features of different gene pools. BMC Genomics 18:59.

Raghami, M., A.I. López-Sesé, M.R. Hasandokht, Z. Zamani, M.R.F. Moghadam, and A. Kashi. 2014. Genetic diversity among melon accessions from Iran and their relationships with melon germplasm of diverse origins using microsatellite markers. Plant Syst. Evol. 300:139-151.

Roy, A., S.S. Bal, M. Fergany, S. Kaur, H. Singh, A.A. Malik, J. Singh, A.J. Monforte, and N.P.S. Dhillon. 2012. Wild melon diversity in India (Punjab state). Genet. Resources Crop Evol. 59:755-767. 
Seung, Y. and K.S. Yong. 2006. Development of oriental melon (Cucumis melo L.)-derived SSR markers using a PCR-based method and polymorphic application for the genotyping of commercial lines. Korean J. Genet. 28:317324.

Song, H., X. Cui, H. Ma, Z. Zhu, and F. Luan. 2012. Construction of DNA fingerprint database based on SSR marker for varieties (lines) of Cucumis melo L. Zhongguo Nong Ye Ke Xue 45:2676-2689.

Vogler, A.P. and M.T. Monaghan. 2007. Recent advances in DNA taxonomy. J. Zoological Syst. Evol. Res. 45:1-10.
Wang, M.R., Y. Xu, and Y.L. Zhan. 2010. Construction of SSR fingerprint on thick skin melon varieties. Chinese Agr. Sci. Bul. 26:47-51.

Waugh, J. 2007. DNA barcoding in animal species: Progress, potential and pitfalls. BioEssays 29:188-197.

Weng, Y. 2010. Genetic diversity among Cucumis metuliferus populations revealed by cucumber microsatellites. HortScience 45:214-219.

Yang, N., Y.Z. Sun, and J.X. Wu. 2016. Economic analysis of watermelon and muskmelon production and trade. Zhongguo Gua-Cai 29:1-9.

Yuan, T., M. He, and L. Huang. 2009. Intraspecific genetic variation in mitochondrial $16 \mathrm{~S}$ rRNA and
COI genes in domestic and wild populations of huaguizhikong scallop chlamys nobilis reeve. Aquaculture 289:19-25.

Zhang, Y.B., X.B. Fan, Y. Sh. Aierken, X.L. Ma, H.P Yi, and M.Z. Wu. 2017. Genetic diversity of melon landraces (Cucumis melo L.) in the Xinjiang Uygur Autonomous Region on the basis of simple sequence repeat markers64:10231035.

Zhu, H., L. Guo, P. Song, F. Luan, J. Hu, X. Sun, and L. Yang. 2016. Development of genomewide SSR markers in melon with their crossspecies transferability analysis and utilization in genetic diversity study. Mol. Breed. 36:153. 